\title{
Paget-Schroetter Syndrome Resulting from Thoracic Outlet Syndrome and KAATSU Training
}

\author{
Tatsunori Noto, Go Hashimoto, Takahito Takagi, Toru Awaya, Tadashi Araki, \\ Masanori Shiba, Raisuke Iijima, Hidehiko Hara, Masao Moroi, \\ Masato Nakamura and Kaoru Sugi
}

\begin{abstract}
:
A 29-year-old woman who worked as a KAATSU (a type of body exercise that involves blood flow restriction) instructor visited our emergency room with a chief complaint of swelling and left upper limb pain. Chest computed tomography (CT) showed non-uniform contrast images corresponding to the site from the left axillary vein to the left subclavian vein; vascular ultrasonography of the upper limb revealed a thrombotic obstruction at the same site, leading to a diagnosis of Paget-Schroetter syndrome (PSS). We herein report our experience with a case of PSS derived from thoracic outlet syndrome (TOS), in a patient who was a KAATSU instructor.
\end{abstract}

Key words: Paget-Schroetter syndrome, KAATSU training, thoracic outlet syndrome

(Intern Med 56: 2595-2601, 2017)

(DOI: 10.2169/internalmedicine.7937-16)

\section{Introduction}

Vascular endothelial dysfunction is induced in patients with Paget-Schroetter syndrome (PSS) by the retraction of the veins passing the thoracic outlet due to the development of the upper limb muscles. This retraction eventually leads to thrombus formation $(1,2)$. It is considered to be caused by excessive outer rotation exercise that retracts the veins, particularly in young people who frequently exercise their upper limbs (3). PSS, which has an annual incidence of 2 per 100,000 population, is an extremely rare disease (4).

KAATSU training is a type of muscle training that is performed under blood flow restriction by applying appropriate pressure to the base of the upper limbs or thighs using a pressurization belt. We herein report our experience of a case of PSS derived from thoracic outlet syndrome (TOS), in which the patient was a KAATSU instructor.

\section{Case Report}

The case was a 29-year-old woman. She had been work- ing as a KAATSU instructor for two years. She had no significant medical or family history. However, she had been suffering from localized edema on the left collarbone with mild tenderness for six years. When performing KAATSU training herself, she trained for 30 minutes to 1 hour, approximately 3 times a week. During training, she usually kept constant pressure on her upper limbs, and in some cases, she continued to apply the pressure for an hour. She became aware of swelling, pain, and discoloration of her left upper limb during KAATSU training - she visited her local doctor after the symptoms persisted for approximately six days. A detailed examination was scheduled, but due to the severity of her symptoms, she visited our emergency room as an outpatient the following day. A physical examination revealed the following: height, $165 \mathrm{~cm}$; body weight, $49 \mathrm{~kg}$; body mass index, $20 \mathrm{~kg} / \mathrm{m}^{2}$; blood pressure, $114 / 78 \mathrm{mmHg}$;

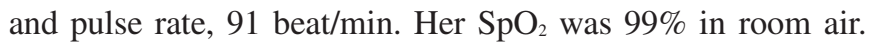
She had clear breathing sounds and normal heart sounds. She complained of swelling and pressure pain in her left upper limb and showed dark-red discoloration without edema in her lower limbs. A blood test revealed a slight increase in her D-dimer $(1.7 \mu \mathrm{g} / \mathrm{mL})$ level. No antiphospholipid anti- 
Table. Blood Test.

\begin{tabular}{lrl}
\hline WBC & 6,500 & $/ \mu \mathrm{L}$ \\
RBC & 408 & $\times 10^{6} / \mu \mathrm{L}$ \\
$\mathrm{Hb}$ & $12.1 \mathrm{~g} / \mathrm{dL}$ \\
$\mathrm{Hct}$ & 36.5 & $\%$ \\
Plt & 31.2 & $10^{4} / \mu \mathrm{L}$ \\
T-CHO & 133 & $\mathrm{mg} / \mathrm{dL}$ \\
TG & 66 & $\mathrm{mg} / \mathrm{dL}$ \\
$\mathrm{HDL}$ & $55 \mathrm{mg} / \mathrm{dL}$ \\
LDL & $72 \mathrm{mg} / \mathrm{dL}$ \\
PT(\%) & 66 & $\%$ \\
PT(INR) & 1.20 \\
FIB & $215 \mathrm{mg} / \mathrm{dL}$ \\
D-D & 1.3 & $\mu \mathrm{g} / \mathrm{mL}$ \\
AT-III & 113 & $\mu \mathrm{g} / \mathrm{mL}$ \\
PS Ag & 90 & $\%$ \\
PS Activity & $76 \%$ \\
PC Ag & $78 \%$ \\
PC Activity & $79 \%$ \\
ANA & - & \\
aCL & $<1.2$ & $\mathrm{U} / \mathrm{mL}$ \\
LA & 1.08 \\
\hline
\end{tabular}

Blood tests showed a slight increase in the D-dimer levels $(1.7 \mu \mathrm{g} / \mathrm{mL})$. No activation of the antiphospholipid antibodies was observed. In addition, there was no decrease in the levels of protein $\mathrm{C}$ or protein $\mathrm{S}$.

PS: protein S, PC: protein C, ANA: antinuclear antibody, aCL: anticardiolipin antibody, LA: lupus anticoagulant

body activation was observed and there was no decrease in her protein $\mathrm{C}$ or protein $\mathrm{S}$ levels (Table. 1). A chest X-ray showed a cardiothoracic ratio of $36 \%$ with a dull right costophrenic angle (Fig. 1). An electrocardiogram revealed normal sinus rhythm with no right heart strain (Fig. 2). Chest computed tomography (CT) showed non-uniform contrast images corresponding to the site from the left axillary vein to the left subclavian vein. A tubercular shadow due to lung infarction was observed at the base of the right lung (Fig. 3). Vascular ultrasonography of the upper limbs showed a thrombus of non-uniform brightness from the left axillary vein to the bifurcation of the left brachiocephalic vein and the left internal jugular vein, along with a mobile 3 $\mathrm{mm} \times 3 \mathrm{~mm}$ thrombus on the central side (Fig. 4). Lung perfusion scintigraphy revealed large numbers of perfusion defects in both lungs, which were determined to be complications of pulmonary thromboembolism (Fig. 5). However, cardiac ultrasonography revealed no evidence of pulmonary hypertension or right atrial or ventricular dilatation (Fig. 6).

Anticoagulation treatment consisting of the continuous infusion of unfractionated heparin and warfarin was initiated upon the diagnosis of left subclavian vein thrombosis. While her symptoms showed a trend toward improvement following the initiation of treatment, the left upper limb pain that she experienced on elevation and abduction showed no im-

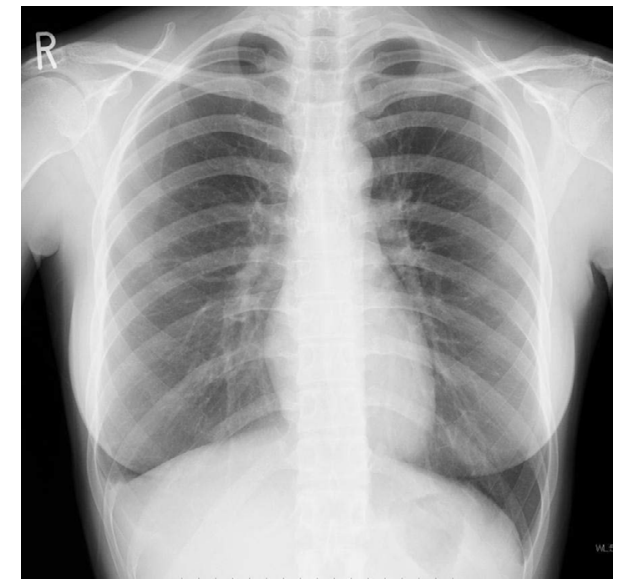

Figure 1. Chest X-ray. The chest X-ray showed a cardiothoracic ratio of $36 \%$ with a dull right costophrenic angle.

provement. Thrombectomy by catheter was considered; however, due to the risk of developing additional pulmonary thromboembolisms and the fact that a partially organized thrombus was observed, complete removal was considered difficult, and was not performed. From day 14 of her illness, thrombolytic therapy (intravenous urokinase, 240,000 units/ day) was administered for 8 days. As a result, the swelling and discoloration of the left upper limb improved (Fig. 7). Follow-up vascular ultrasonography of the upper limb showed the shortening of the thrombus and recanalization of the blood flow on the sixth day of treatment. The mobile thrombus disappeared, and the thrombus shortened. Newer blood flow was observed. The D-dimer levels gradually decreased to a normal level without evanescent elevation after the start of anticoagulation therapy (Fig. 8). Follow-up chest CT showed a narrowing of the space between the left collarbone and the first rib when elevating the upper limb (Fig. 9).

The patient was diagnosed with PSS after we identified TOS on CT. We suspected that the TOS and KAATSU training had caused the patient's PSS. The patient was discharged from the hospital on Day 25 of her illness. Internal warfarin treatment was continued after discharge. She quit KAATSU training after discharge. Follow-up vascular ultrasonography of her left upper limb at approximately one year from discharge still showed blood flow signals; however, these signals were nearly interrupted in her left subclavian vein when she elevated her left upper limb (Fig. 10). However, her clinical course is good. She has stated a desire to bear children, so she discontinued internal warfarin in the third year after discharge. No relapse of her symptoms has been recognized.

\section{Discussion}

Deep vein thrombosis (DVT) in the upper limb accounts for $4-11 \%$ of all DVT cases $(5,6)$; its major cause is central venous catheter placement. PSS is an idiopathic subclavian vein thrombosis that develops in relatively young people due 


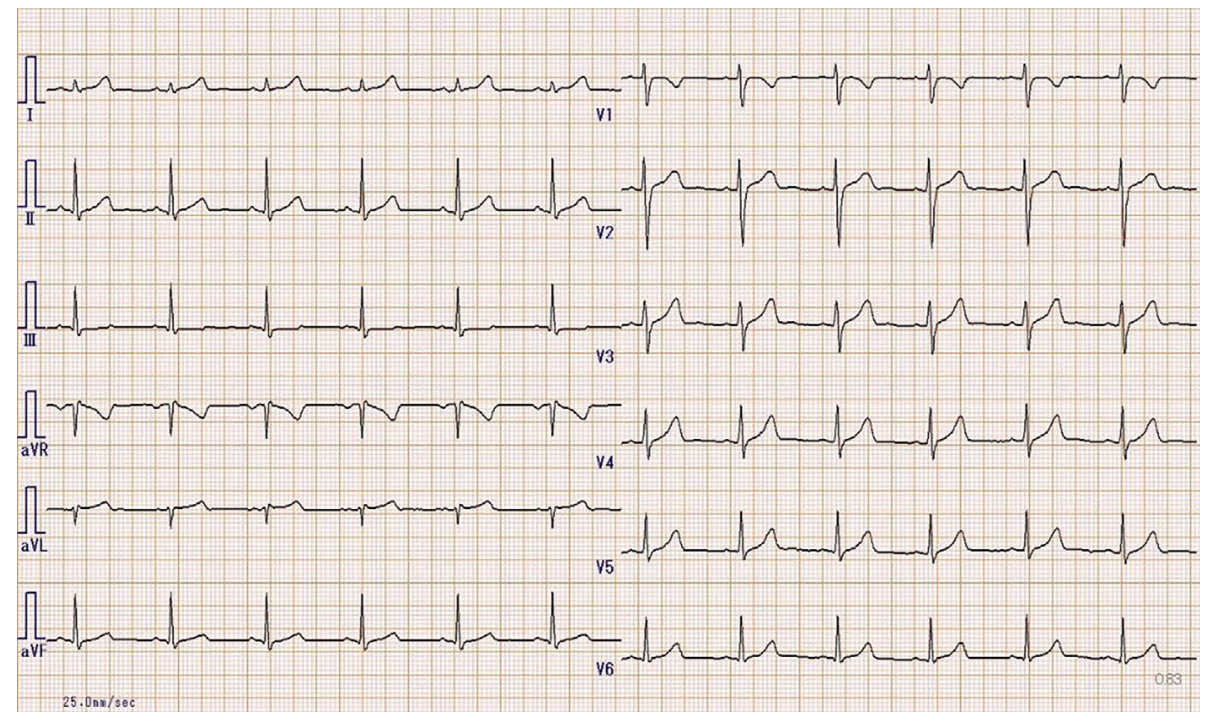

Figure 2. Electrocardiography. The electrocardiogram showed normal sinus rhythm with no sign of right heart strain.

A

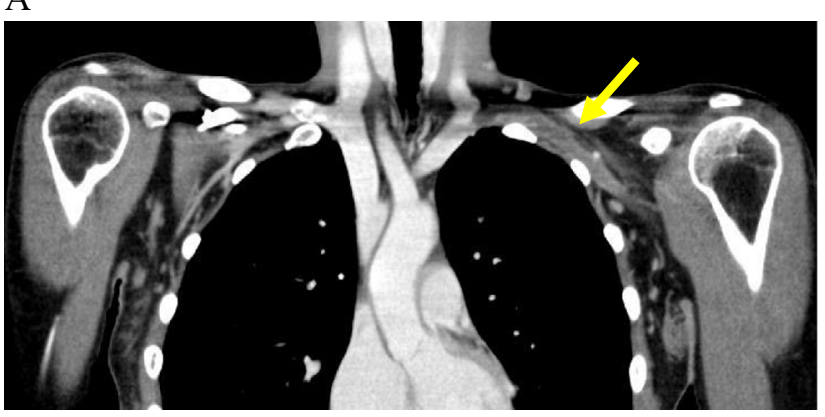

B

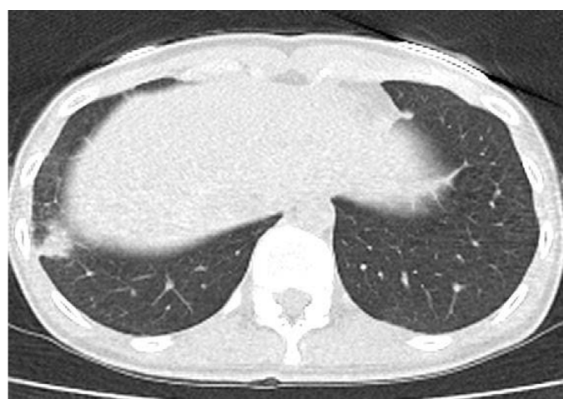

Figure 3. Chest CT. A: Non-uniform contrast images corresponding to the site from the left axillary vein to the left subclavian vein. B: A tubercular shadow due to lung infarction at the base of the right lung.

A

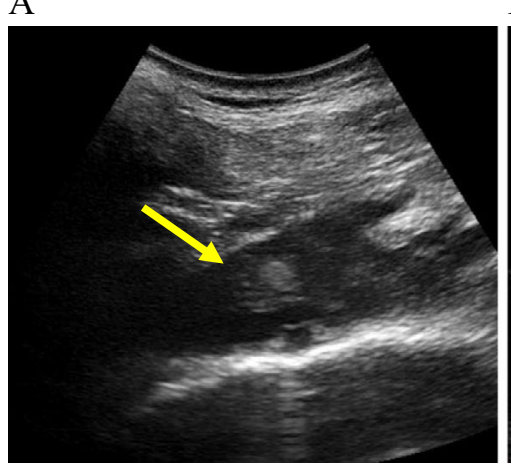

B

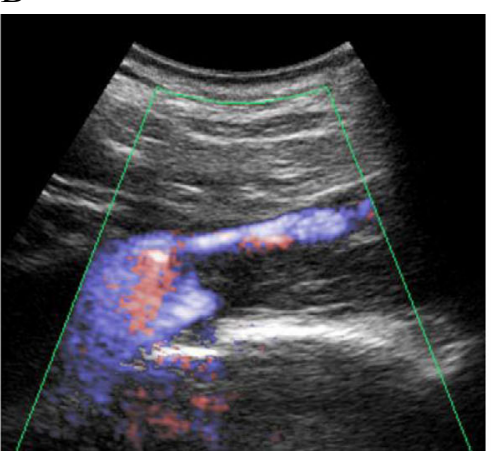

C

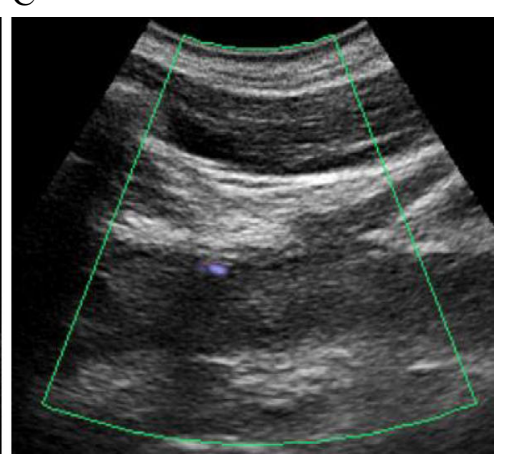

Figure 4. Vascular ultrasonography of the upper limb. A: A mobile $3 \mathrm{~mm} \times 3 \mathrm{~mm}$ thrombus on the central side of the subclavian vein. B: Thrombotic occlusion in the subclavian vein. C: Thrombotic occlusion at the joint of the axillary vein and subclavian vein.

to compression at the thoracic outlet; as such, PSS is included in the broad definition of TOS $(7,8)$. PSS is believed to develop due to retraction of the subclavian vein as a result of excessive exercise of the upper limbs. The retraction of the subclavian vein encourages vein endothelial disorders and thrombus formation $(9,10)$. Thus, PSS is known as "effort thrombosis". Previous reports have shown that the training of the upper limbs by methods such as performing weight-bearing exercises or push-ups can cause PSS $(11,12)$. In the present case, the costoclavicular space 


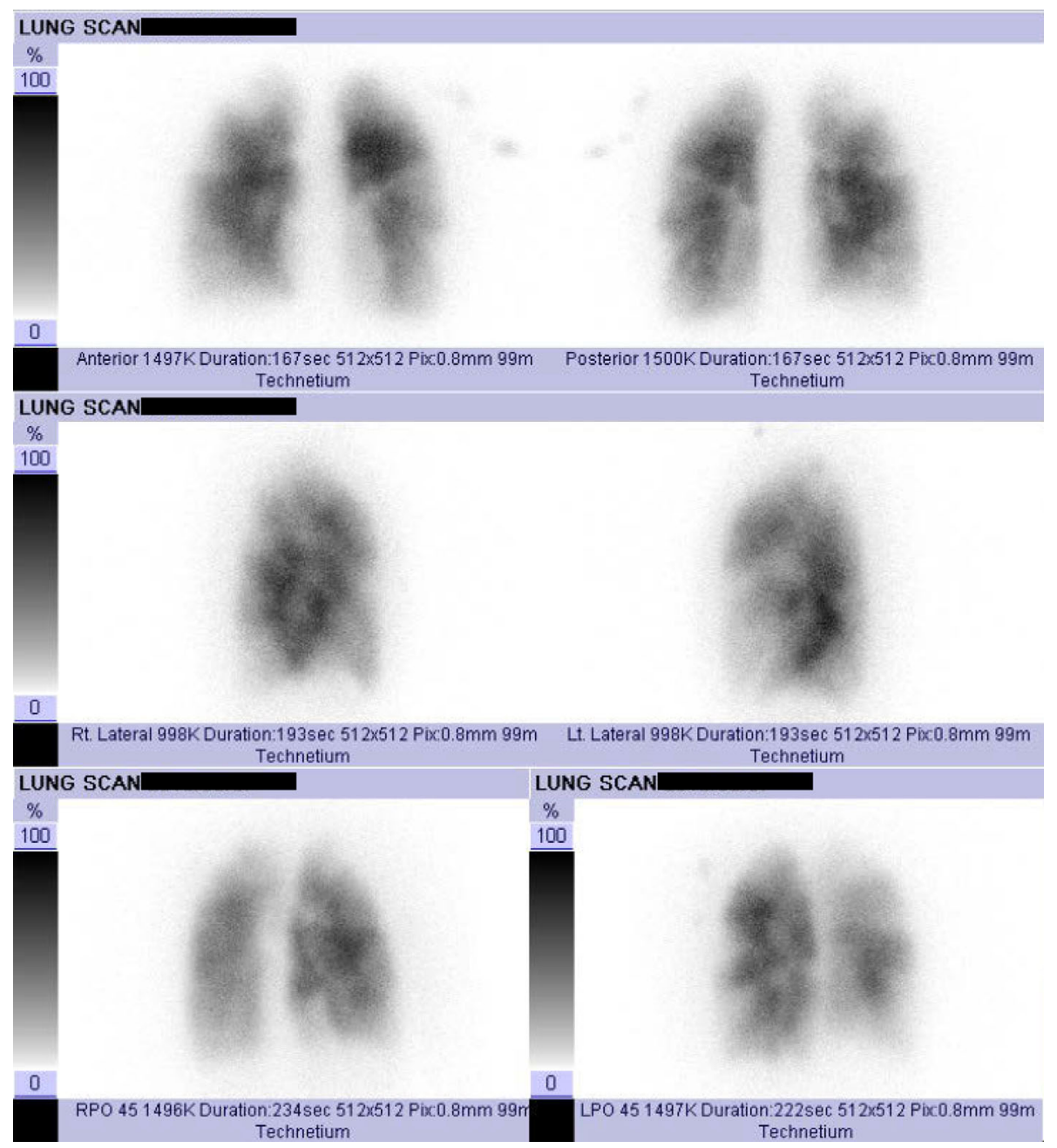

Figure 5. Lung perfusion scintigraphy. Large numbers of perfusion defects were observed in both lungs.

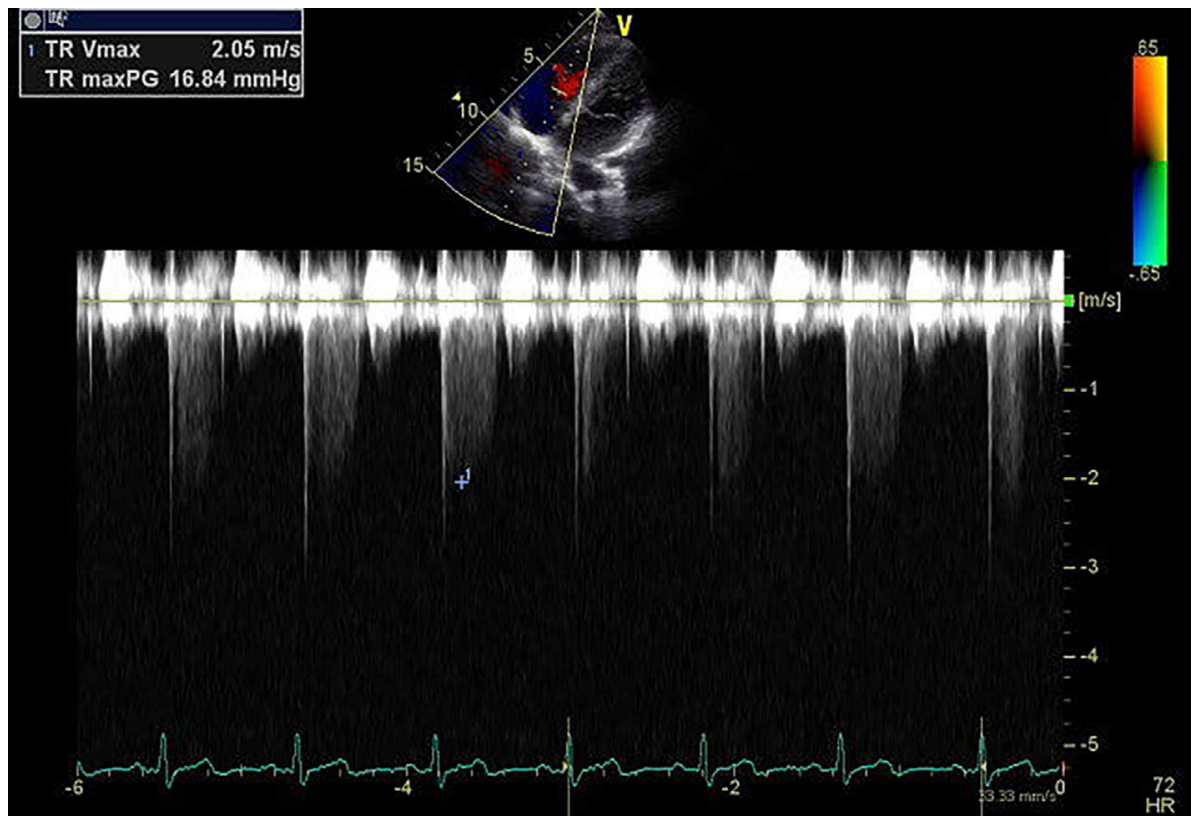

Figure 6. Cardiac ultrasonography. Cardiac ultrasonography showed no evidence of pulmonary hypertension or right atrial or ventricular dilation. 
A

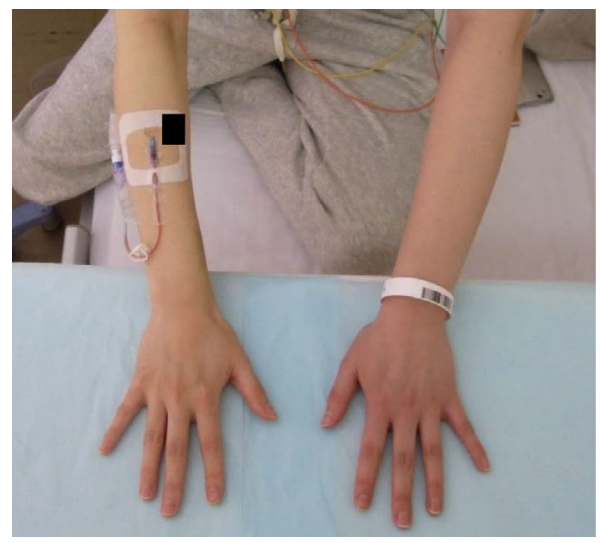

B

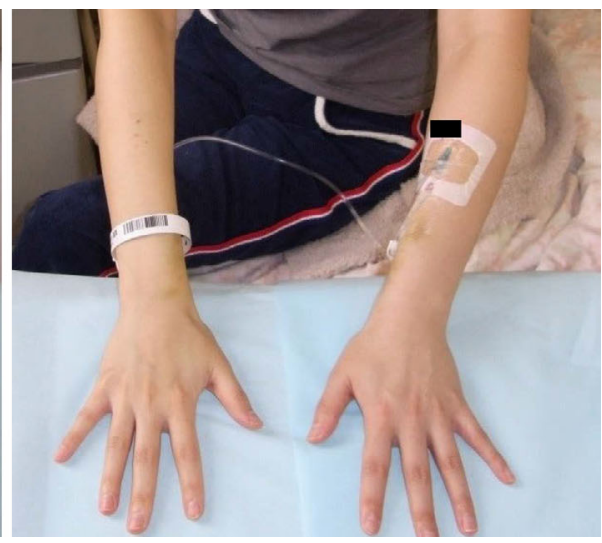

Figure 7. The color of the upper limb before and after treatment. A: Before treatment. The left upper limb was swollen, and the color of the left forearm was dark-red. B: After treatment. The swelling of the left upper arm remained, but the color had improved.

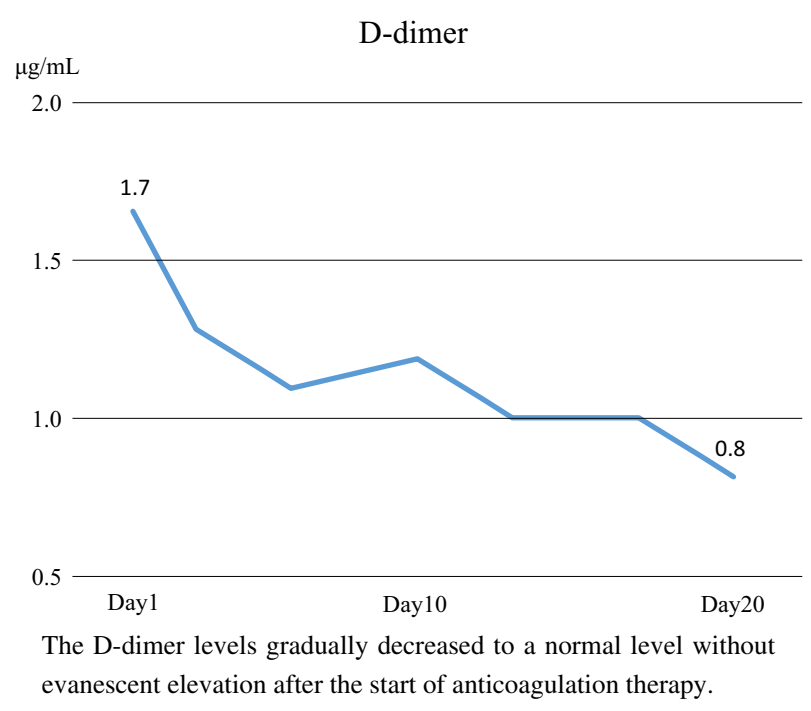

Figure 8. The serial changes in the D-dimer levels.

became narrow when the patient elevated her upper limbs on follow-up chest CT, and the blood flow signals were nearly interrupted in the left subclavian vein when she elevated her left upper limb. We therefore suspected that left subclavian vein thrombosis of TOS origin (i.e. PSS) had developed. Along with the fact that the proximal site of the thrombus reached the subclavian vein, TOS was believed to have contributed to the development of the thrombus.

In the present case, the patient was a KAATSU instructor. KAATSU is a type of muscle training that involves the restriction of blood flow by applying appropriate pressure to the base of the upper limbs or thighs using a pressurization belt. This limited blood flow then supposedly causes accumulation of lactic acid in the muscle. Then, when the belt is removed, hypophysis occurs to increase the amount of lactic acid in the blood, thereby leading to the production of growth hormones and ultimately an increase in muscle mass. During training, the pressure on the upper limbs should be within $100 \mathrm{mmHg}$, and the training should be finished

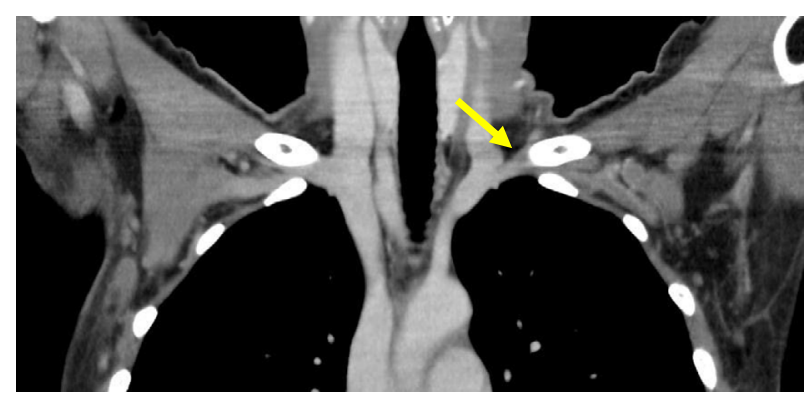

Figure 9. Follow-up chest CT. Follow-up chest CT showed a narrowing of the space between the left collarbone and the first rib when elevating the upper limb.

within 10 minutes per session. However, the present patient usually maintained constant pressure on her upper limbs for an hour.

Her only other complaint was localized edema on the left collarbone with mild tenderness, which she had suffered for six years. She had no other symptoms, such as supination pain or numbness due to TOS when elevating the left upper limb. Although she had been a KAATSU trainer for two years, the localized edema on her left collarbone had been recognized earlier and is considered to have occurred due to TOS. The fact that thrombolytic therapy was not effective in increasing her D-dimer levels and that a thrombus of nonuniform brightness was found by vascular ultrasonography of the upper limbs also suggests that the patient had chronic-rather than acute-thrombosis. This case is considered to have occurred due to the two factors: venous retraction and endothelial dysfunction of the left subclavian vein, which was brought about by TOS; and the stagnation of the blood flow due to the pressure applied during KAATSU training.

In terms of lung infarction, neither electrocardiography nor cardiac ultrasonography revealed any remarkable findings with regard to the right ventricular load. Fortunately, the upper limb vein thrombosis was found before a pulmo- 


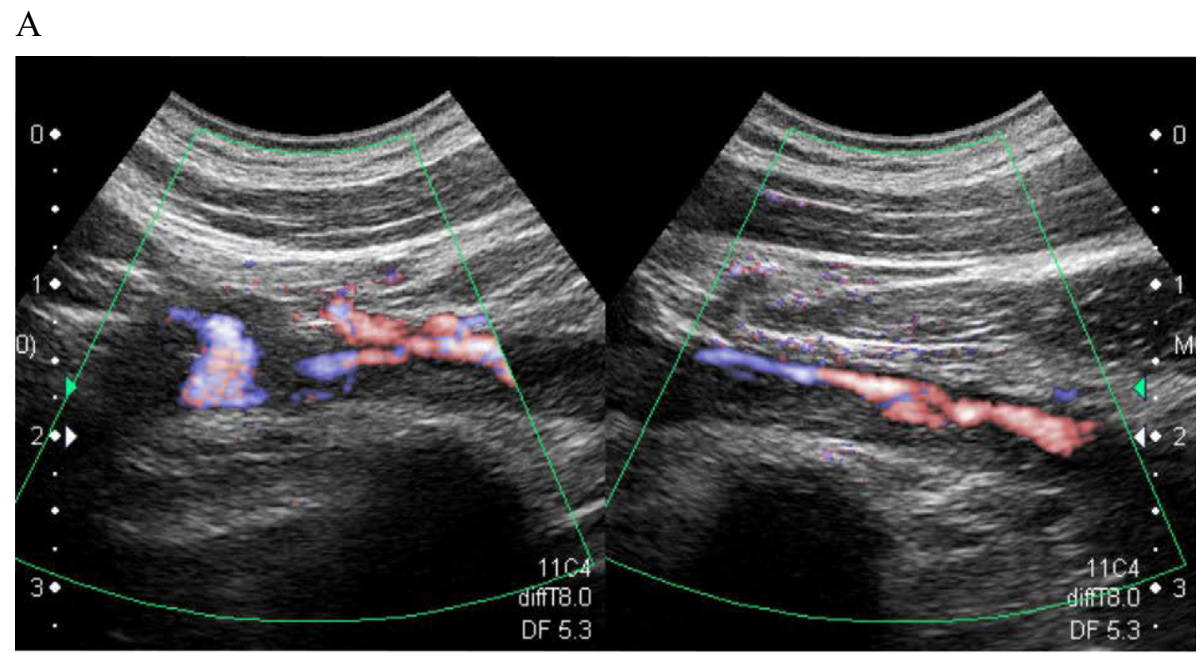

B

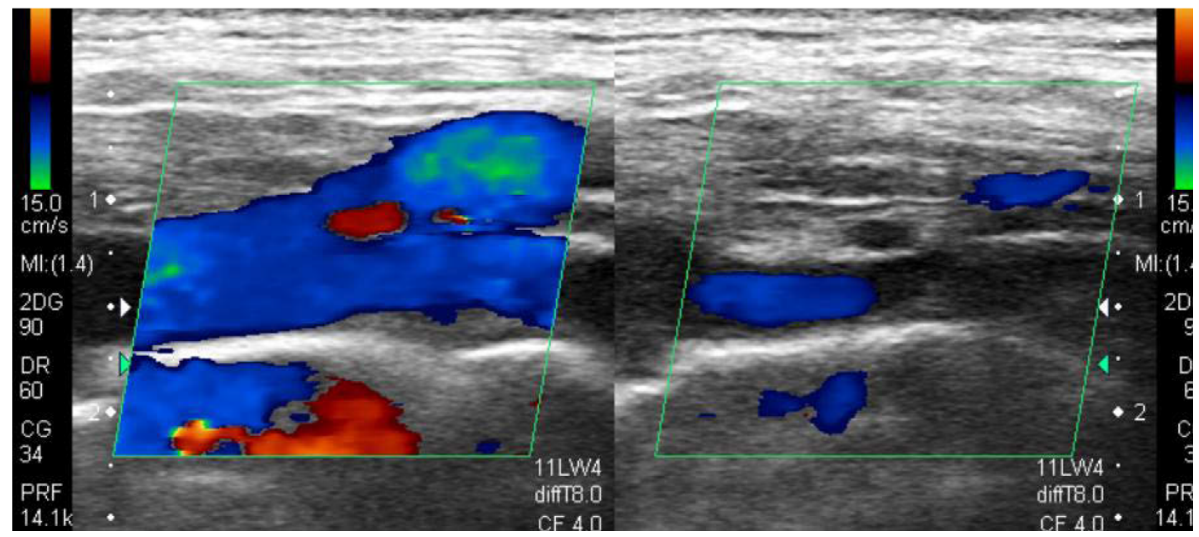

Figure 10. Follow-up vascular ultrasonography of the upper limb. A: Follow-up vascular ultrasonography of her left upper limb showed shortening of the thrombus and recanalization of the blood flow on the sixth day of treatment. The mobile thrombus disappeared, and the thrombus shortened. Newer blood flow was observed. B: Follow-up vascular ultrasonography of her left upper limb at one year after discharge showed that the blood flow signals were nearly interrupted in her left subclavian vein when elevating the left upper limb.

nary thromboembolism developed.

Although no guidelines exist for treating DVT of the upper limbs, the guidelines for pulmonary thromboembolism due to DVT of the lower limbs indicate that anticoagulation therapy with warfarin should be administered for 3-6 months for primary cases with no risk factors, or for as long as the risk continues for relapsed cases or cases with continuing risk factors. A 5-year follow-up report on patients who received warfarin coagulation monotherapy revealed that while $50 \%$ of the cases were asymptomatic, the upper limb symptoms of $13 \%$ of the patients worsened (13). In contrast, according to a report on the administration of anticoagulation and thrombolytic therapy, $76 \%$ of the cases were asymptomatic at 55 months (14), indicating that the combination therapy was associated with an improved outcome. In the present case, the recanalization of the blood flow was observed by conservative therapy alone (the combination of anticoagulation therapy and thrombolytic therapy), and no re-occlusion or recurrence of upper limb symptoms were ob- served under the continuous internal administration of warfarin. However, since thromboembolism was observed as a complication and the thrombus remains, careful follow-up should be continued; in the event of an exacerbation of the thrombus or a recurrence of symptoms, surgical decompression by resection of the first rib should be considered in order to exclude risk factors.

In the present case, the long-term duration of blood flow restriction was likely to have contributed to the development of venous thrombosis. Thus, TOS and KAATSU training were the risk factors that led to thrombus formation. People at risk of thrombosis should be careful when performing KAATSU training. Before performing KAATSU training, people taking oral contraceptives, or who have congenital coagulation defects, should have a detailed understanding of the mechanisms underlying the training and appropriate training methods. The present patient quit KAATSU training and discontinued warfarin in the third year after discharge. However, no relapse of her symptoms has been seen thus 
far.

Aside from the present case, there have been no other reports of KAATSU training causing PSS in patients with TOS. This case shows that overtraining may increase the complications in patients who are at a high risk of thrombosis, such as those with TOS. To prevent complications, KAATSU training should be carried under appropriate supervision. In the present case, we believe that PSS was derived from both TOS and KAATSU training.

The authors state that they have no Conflict of Interest (COI).

\section{References}

1. Paget J. Clinical Lectures and Essays. Longmans Green, and Co, London, 1875.

2. Von Schroetter L. Erkrankungen der Gefasse. In: Nathnagel Handbuch der Pathologie und Therapie. Holder, Vienna, 1884.

3. Won CW, Chang HC, Low CO. Paget-Shroetter syndrome: case report and review of the literature. Internet J Orthoped Surg 2: 1215, 2004.

4. Lindblad B, Tengborn L, Bergqvist D. Deep vein thrombosis of the axillary-subclavian veins: epidemiologic data, effects of different types of treatment and late sequelae. Eur J Vasc Surg 2: 161165, 1988.

5. Joffe HV, Kucher N, Tapson VF, Goldhaber SZ. Upper-extremity deep vein thrombosis: a prospective registry of 592 patients. Circulation 110: 1605-1611, 2004.

6. Muñoz FJ, Mismetti P, Poggio R, et al. Clinical outcome of pa- tients with upper-extremity deep vein thrombosis: results from the RIETE Registry. Chest 133: 143-148, 2008.

7. Andres S, Louis MM. Thoracic Outlet Syndrome: Venous. In: Rutherford's Vascular Surgery. 7th ed. Cronenwett JL, Johnston KW, Eds. Saunders Elsevier, Philadelphia, 2010: 1907-1917.

8. Melby SJ, Vedantham S, Narra VR, et al. Comprehensive surgical management of the competitive athlete with effort thrombosis of the subclavian vein (Paget-Schroetter syndrome). J Vasc Surg 47: 809-821, 2008.

9. Stevenson IM, Parry EW. Radiological study of the aetiological factors in venous obstruction of the upper limb. J Cardiovasc Surg (Torino) 16: 580-585, 1975.

10. Molina JE. Surgery for effort thrombosis of the subclavian vein. J Thorac Cardiovasc Surg 103: 341-346, 1992.

11. Spencer TR, Lagace RE, Waterman G. Effort thrombosis (PagetSchroetter syndrome) in a 16-year-old male. Am J Case Rep 15: 333-336, 2014.

12. Toya N, Fujita T, Ohki T. Push-up exercise induced thrombosis of subclavian vein in a young woman report of a case. Surg Today 37: 1093-1095, 2007.

13. Heron E, Lozinguez O, Emmerich J, et al. Long-term sequelae of spontaneous axillary-subclavian venous thrombosis. Ann Intern Med 131: 510-513, 1999.

14. Lee JT, Karwowski JK, Harris EJ, et al. Long-term trombotic recurrence after nonoperative management of Paget-Schroetter syndrome. J Vasc Surg 43: 1236-1243, 2006.

The Internal Medicine is an Open Access article distributed under the Creative Commons Attribution-NonCommercial-NoDerivatives 4.0 International License. To view the details of this license, please visit (https://creativecommons.org/licenses/ by-nc-nd/4.0/).

(C) 2017 The Japanese Society of Internal Medicine Intern Med 56: 2595-2601, 2017 